\title{
IMPROVED HEAT TRANSFER PREDICTION ENGINEERING CAPABILITIES FOR ROCKET THRUST CHAMBER LAYOUT
}

\section{Maeding, D. Wiedmann, K. Quering, and O. Knab}

Astrium GmbH Space Transportation Launcher Propulsion, System Analysis Munich 81663, Germany

The demand for a more comprehensive engineering tool for design and parametric investigations of thrust-chamber relevant heat transfer is pushing the improvement of coolant and hot gas side prediction tools. Regenerative Coolant Flow Simulation (RCFS) [1], Astrium in-house developed one-dimensional (1D) tool to compute hot gas and coolant side heat transfer in a coupled approach, is based on the hot gas side Cinjarew approach which has its origin in the late 1960s. This tool was used as a starting basis for the development and validation of a further improved method. Over the past years, Astrium Space Transportation (ST) has continuously expanded the knowledge in this field. In addition, subscale hot firings, using different propellant combinations and injection conditions, relevant to open and closed cycle applications, were used for the second RCFS generation - the RCFS-II.

\section{INTRODUCTION}

The present propulsion development is more and more marked by a transition from a pure experimental work to a reasonable share between experimental work and computational design investigation. This approach requires adequate design tools covering all phases of engine development. The modern thrust chamber design work at Astrium ST, including thrust chamber sizing, hydraulic, heat transfer, and combustion analysis, is based on a combination of commercial and in-house tools. The commercial CFX, the in-house Rocflam-II (Rocket Flow Analysis Module) [2] and the RCFS-II codes form the main three pillars for a successful thrust chamber design in terms of combustion and heat transfer [3].

This is an Open Access article distributed under the terms of the Creative Commons Attribution-Noncommercial License 3.0, which permits unrestricted use, distribution, and reproduction in any noncommercial medium, provided the original work is properly cited. 


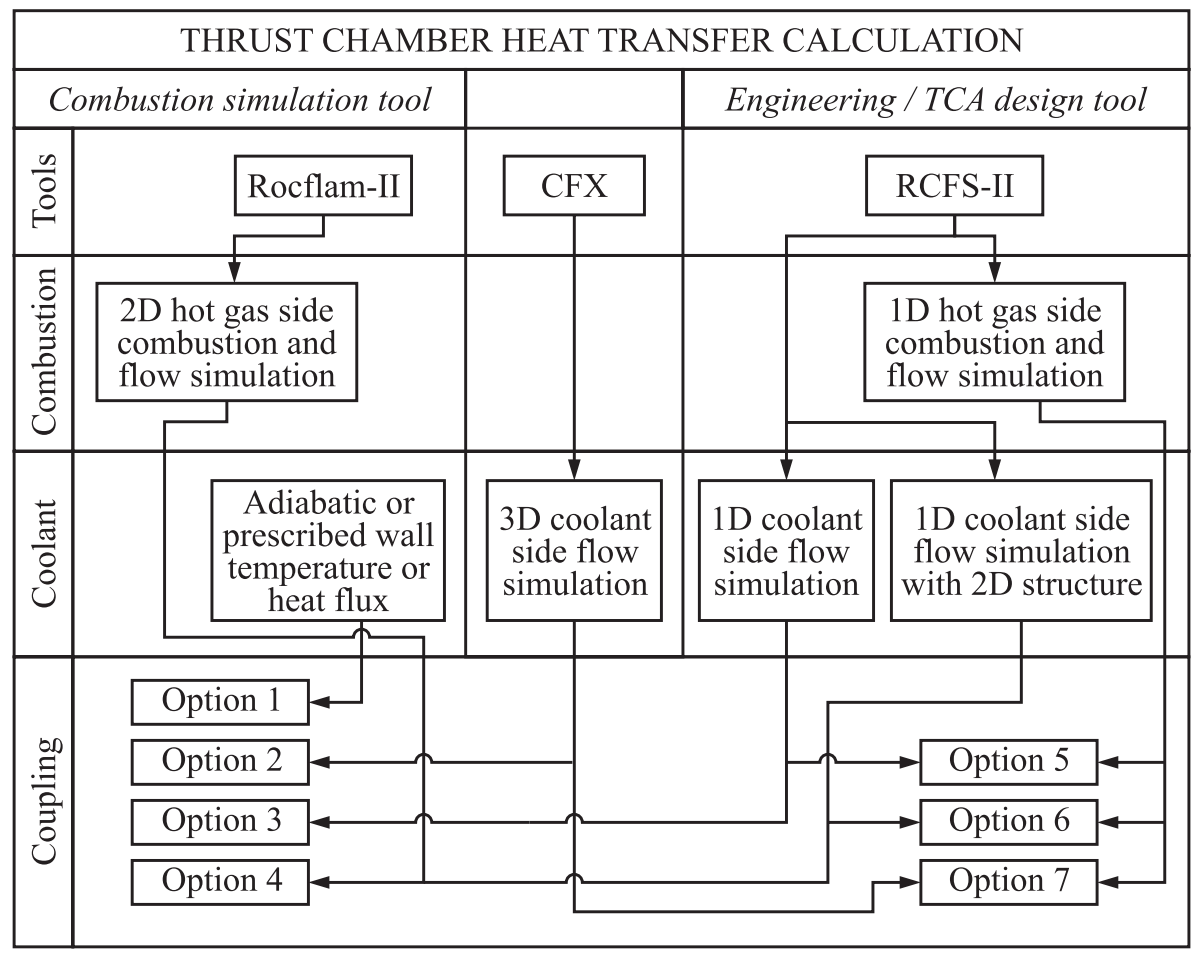

Figure 1 Rocket combustion and heat transfer simulation tools

Special interfaces between the different tools allow performing integrated sensitivity analyses using different tools for hot gas and coolant side simulations. Significant advantage is the direct transferability of the cooling channel geometry, established by RCFS-II layout calculations, towards the two-dimensional (2D) Rocflam-II combustion code simulation as well as the three-dimensional (3D) CFX coolant side heat transfer computation - presenting a high end heat transfer, combustion and hydraulic analysis approach. The possible coupling combinations of the three different tools are presented in Fig. 1.

Rocflam-II presents a 2D axisymmetric, finite volume Navier-Stokes code with an implemented chemical reaction model and parallel particle tracking. The reaction mechanism of bi-propellant systems can be approximated by a certain number of species. The tool allows simulating multiple discrete phases and computing the trajectories of these discrete phase entities by integrating their force balance taking into account various topics.

The subject of the paper is to highlight the main new features of Astrium's improved heat transfer RCFS-II engineering tool as well as the general approach 
used to develop and to verify this new method. One of the major development and improvement objectives is the still ongoing comparison of the calculation results with hot run test data in order to determine the degree of prediction accuracy and to determine the further direction of tool improvement.

\section{GENERAL HEAT TRANSFER APPROACH}

At the early design and development phase of a new thrust chamber, an efficient simulation tool is needed to cover the main features of the new system without having test data available. The RCFS-II presents an upgraded version of the well-known RCFS code [1]. In order to meet the growing requirements in terms of reliability and also with respect to new propellant combinations like $\mathrm{LOx}-\mathrm{CH}_{4}$, significant improvements were required. The RCFS code, used for many years, was a hot-gas side Nusselt $(\mathrm{Nu})$ correlation based method (Cinjarew approach), reflecting all systems developed and used within Astrium ST. However, this $1 \mathrm{D}$ approach required a huge data base to reflect real system behavior, due to the drawbacks of the pure $\mathrm{Nu}$-correlation and different implemented empirical factors.

Over the past years, Astrium ST has continuously expanded the knowledge in this field. In addition, subscale hot firings, using different propellant combinations ( $\mathrm{LOx}-\mathrm{H}_{2}, \mathrm{LOx}-\mathrm{CH}_{4}$, and $\mathrm{LOx}-$ kerosene) and injection conditions, relevant to open and closed cycle applications, were used. The application of the initial RCFS code revealed a number of inadequacies, which were covered by applying empirical correlations in order to match realistic conditions. To meet these requirements and to increase the prediction accuracy already during an early state of thrust chamber development, a higher-level sophisticated hot-gas side heattransfer calculation method was implemented in the second generation RCFS code, the RCFS-II. One major requirement was to increase the accuracy of the heat transfer prediction with respect to new propellant combinations, such as $\mathrm{LOx}$-kerosene or $\mathrm{LOx}-\mathrm{CH}_{4}$ taking also into account relevant injection conditions.

In the RCFS-II code, a so-called noninteracting core flow — boundary layer (two-stream model) approach is used (no iteration between the potential core flow and the boundary layer). The distribution of main parameters like static pressure, velocity, enthalpy, and gas composition at the edge of the boundary layer is determined by a 1D flow approach throughout the whole thrust chamber, even when a three-stream model with a $2 \mathrm{D}$ wall pressure distribution is used. This means that at $y=\delta_{\mathrm{bl}}$ (edge of the boundary layer), the main parameters correspond to the 1D main flow. The 1D pressure distribution as well as main parameter definition is based on the Gordon-McBride equilibrium com- 


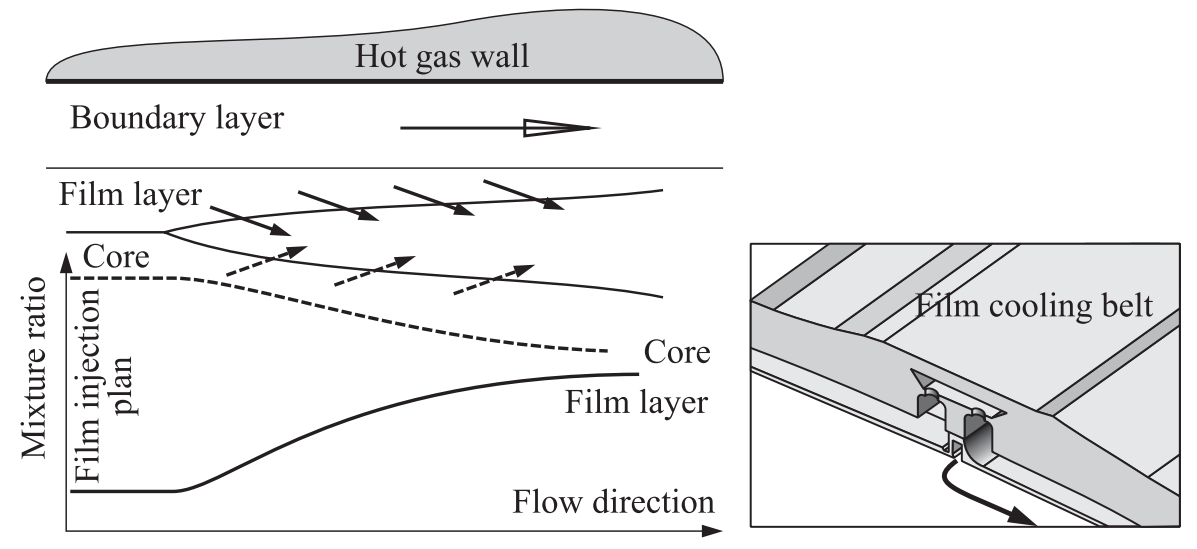

Figure 2 General flow picture if low-temperature film is applied

bustion code $[4,5]$. Within the whole thrust chamber assembly (TCA) and the boundary layer, equilibrium gas composition is used, since the adequate nonequilibrium code is currently not available. The whole picture of losses is covered by considering the combustion efficiency.

All relevant hot-gas parameters at $y=0$ (at the hot gas wall) are calculated also assuming equilibrium combustion and applying $T=T_{\text {wall }}$. The parameter definition, including the chemical composition, at the hot gas wall is made using a $(T, p)$-problem definition file in the Gordon-McBride combustion tool.

The low-temperature layer (film injection or element trimming) between the core and the boundary can be considered by implementing an additional stream between the core and the boundary layer. This requires an adequate mixing model between the core and the film layer, in order to generate the proper conditions for the edge of the boundary layer and relevant heat transfer, assuming a boundary layer mixture ratio equal to that in the low-temperature layer (Fig. 2). The mixing between the two flows is mainly driven by the matching condition between the core and film layer as well as by the turbulence defining the mixing length (effective film length).

By implementing a boundary layer approach for heat transfer calculation, a very important characteristic appears, which is missing in the simple Nucorrelation. An increased cylindrical running length leads to a certain heat flux decrease while propagating downstream the cylinder towards the throat section. The Nu-based heat flux is marked by a constant heat flux along the cylindrical chamber section, neglecting the boundary layer thickness evolution along the chamber cylinder. This thickness is also mainly affected by the shape of the thrust chamber and, therefore, cannot be sufficiently reflected by a $\mathrm{Nu}-$ correlation. 
A major issue, while considering only the molecular motion, typical for the boundary layer approach, is to take into account the additional heat release due to recombination processes in the cold near-wall layer. Therefore, the entire approach is based on the dissociation-free system with heat flux intensification due to a higher hot-gas temperature keeping the core enthalpy unchanged.

The heat flux is determined by the following equation:

$$
q=-\frac{\lambda_{m}+\lambda_{t}}{c_{p}} \frac{\partial h_{\infty}}{\partial y}=\alpha_{\mathrm{turb}} \rho_{\mathrm{av}} v_{\infty}\left(h_{\infty}-h_{\mathrm{wall}}\right)
$$

where $\lambda_{m}$ is the molecular heat conductivity; $\lambda_{t}$ is the turbulent heat conductivity; $c_{p}$ is the average specific heat; $\alpha_{\text {turb }}$ is the dimensionless heat transfer coefficient; $\rho_{\mathrm{av}}$ is the average density; $v_{\infty}$ is the velocity of the boundary layer edge; $h_{\infty}=h+u^{2} / 2$ is the stagnation enthalpy; and $h_{\text {wall }}$ is the enthalpy at the hot gas wall.

The dimensionless heat transfer coefficient needed for heat transfer calculation depends on the temperature and velocity distribution across the boundary layer, assuming that the hydrodynamic boundary layer is thicker than the thermal boundary layer. Therefore, the whole process is driven by Prandtl number $\operatorname{Pr}<1$, which is typical for a compressible flow in rocket chambers.

The implemented model does not consider additional effects like exothermic monopropellant decomposition if Monomethyl Hydrazine (MMH) or Unsymmetrical Dimethylhydrazine (UDMH) film injection is applied. The impact of such additional effects is mainly driven by the reaction time with respect to the overall combustion process and mixing. Only if the monopropellant decomposition rate exceeds the overall rates of mixing and combustion processes, a noticeable change in heat transfer should occur.

The tests with MMH showed that at moderate temperatures of $T$ $=400 \ldots 700 \mathrm{~K}$, monopropellant decomposition processes run very slowly and, therefore, can be neglected as compared to the rates of chemical reactions of the bi-propellants [6].

The current RCFS-II configuration does not also consider the droplet formation and vaporization at injection. In most cases, the propellant injection takes place under supercritical conditions in terms of pressure or temperature leading to a zero surface tension. In this case, the mixing of the injected propellants depends on the degree of turbulence assuming sudden combustion of the mixed propellants, i. e., infinitely small combustion time. This viewpoint leads to a certain simplification, which is significant for the accuracy of the engineering tool. This is true, in particular, for rocket chambers with a low Mach number in the cylindrical section, where the main combustion takes place. The gas propagation time here is higher than the relaxation time to reach a new equilibrium-state gas composition. 


\section{HOT GAS AND BOUNDARY LAYER CONDITIONS: IMPLEMENTED MODULES}

One major issue was to find a solution which permits considering the influence of injector and chamber dependent combustion efficiencies on the heat transfer. Earlier investigations already showed a certain dependence between the completeness of the combustion process and the heat transfer. Due to the absence of a practical method considering the incomplete combustion at local gas composition and taking into account that the nonequilibrium combustion does not consider the overall picture of losses included in the combustion efficiency, another approach was chosen. As a matter of fact, the overall combustion efficiency reflects a combination of boundary, combustion, mixing, and flow field losses without clear differentiation between the contribution of the different factors. This unique solution allows taking into account the expected level of combustion efficiency as an essential heat transfer driver. It became possible to perform sensitivity analyses in terms of combustion efficiency variation.

\subsection{Injector}

The total heat load in the cylindrical chamber section is mainly driven by the injector. The accurate knowledge of heat flux levels in the corresponding chamber region governs the tailoring conditions for the cooling channels to optimize the pressure drop in the regenerative cooling circuit. According to the available in-house experience, the distance between the outer-element row and wall, element type, element size as well as the propellant type and state were identified as the major parameters. In order to solve this issue, an additional method, based on the two-layer mixing model applied to the outer-row injection elements, was implemented eliminating a major problem of the selected boundary layer method which assumes a zero boundary layer thickness at the injector head. Due to the zero boundary layer thickness at the injector head, a very high heat flux arises which assumes that a uniform mixture ratio distribution is already present. In reality, the heat flux at the injector head is mainly determined by the outer-row injection element inducing the mixing process. The mixing of jets is represented by a particular module applying a predefined turbulence degree.

In the case of an open-cycle system, both propellants will be injected under unmixed conditions. Transition to a closed-cycle application implies that a preburned gas with a higher temperature and certain gas composition will be injected, thus affecting the slope of the heat flux at the injector.

The injector module in the current RCFS-II code allows also reflecting the impact of simple coaxial or swirling injection on the heat transfer slope close to the injector head. 


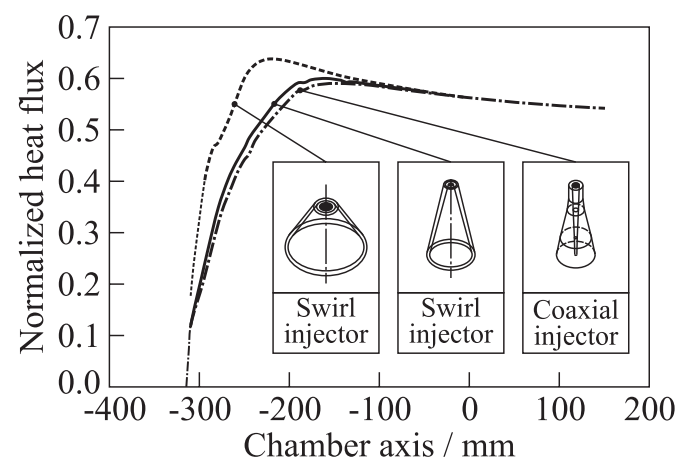

Figure 3 Impact of the injection angle on the heat transfer slope (assuming equal combustion efficiency)

Figure 3 shows the impact of the injection angle in different swirl injectors on the slope of heat transfer. A wide hollow-cone propellant injection leads to a steeper heat flux slope due to more propellant interaction and mixing in radial direction.

Transition from a pure, cold bi-propellant injection to a mixed coldpreburned propellant injection, typical for staged combustion applications leads to a fast heat flux increase close to the injector head due to a higher average injection temperature, keeping the average injection angle unchanged.

Figure 4 shows the impact of staged combustion like bi-propellant injection using the same type of injection element.

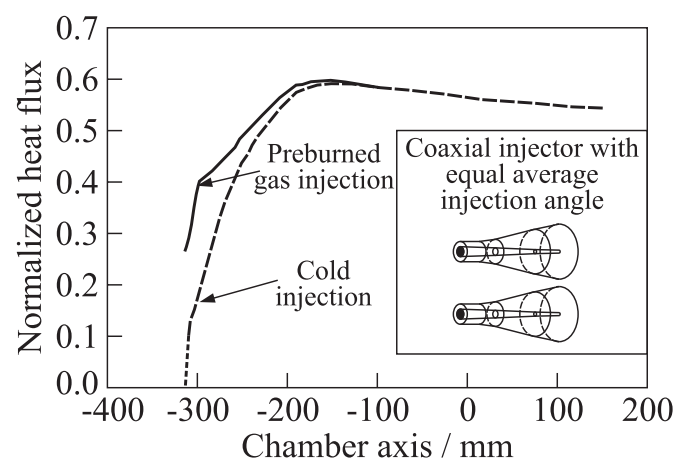

Figure 4 Heat flux evolution (assuming equal combustion efficiency) for cold and preburned propellant injection 


\subsection{Two-Dimensional Nozzle Expansion}

Different analyses of the new RCFS-II code revealed the shortcoming of the 1D boundary layer approach for the supersonic nozzle section. In particular, strong change in the wall pressure in the parabolic nozzle consisting of an arc and a parabola leads to a strong change in the heat flux. Therefore, a 2D wall pressure distribution approach was implemented providing a more realistic hot gas side heat transfer distribution.

The 2D wall pressure distribution, based on TDK [7] or Rocflam-II calculations can be used in order to properly resolve the specific near-wall flow. The corresponding hot gas properties are calculated using a modified Gordon-McBride multistream approach dividing the flow into three main streams (shown schematically in Fig. 5):

$-1 \mathrm{D}$ core flow;

- edge flow of the potential flow field; and

- boundary layer flow.

The consideration of the $2 \mathrm{D}$ wall pressure takes into account the changed core flow behavior marked by a higher velocity in the near-wall layer just after

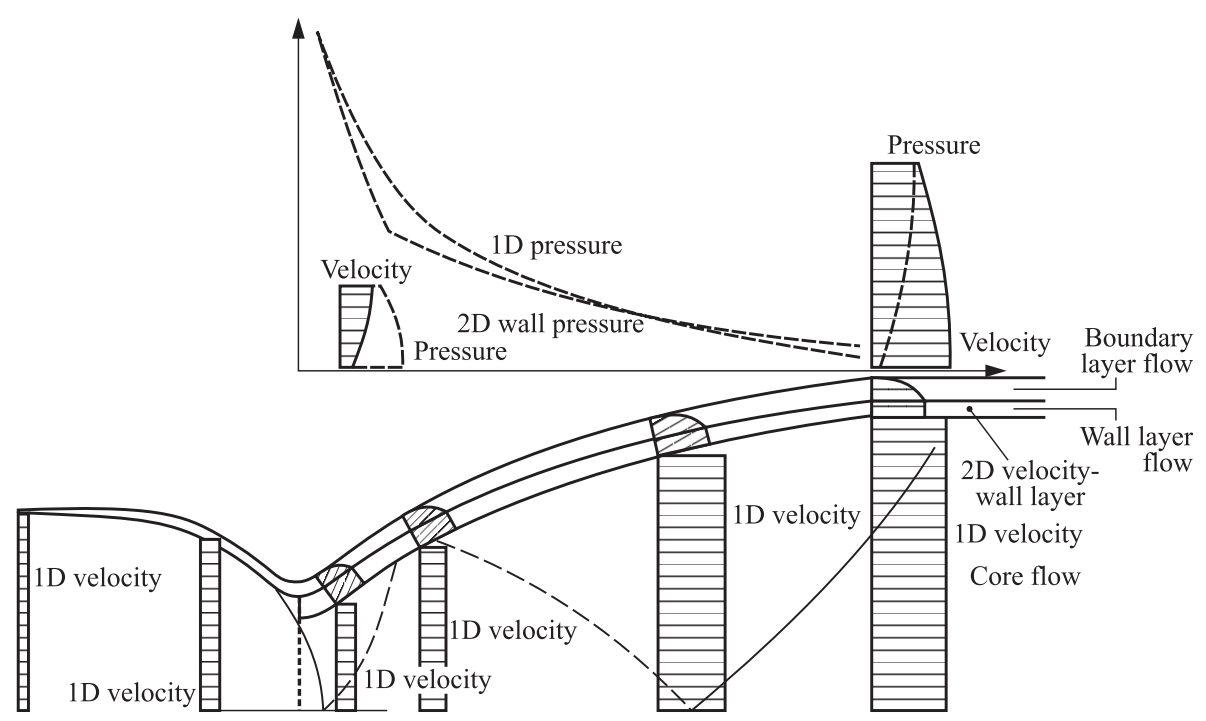

Figure 5 Two- and three-stream models to reflect the 2D supersonic flow effect on the heat flux to the wall (wall layer flow - TDK based pressure data) 


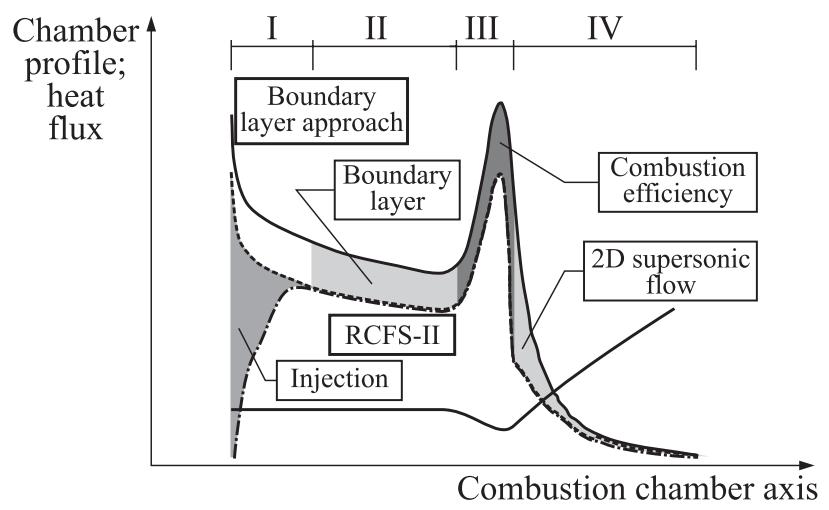

Figure 6 Visualization of implemented RCFS-II heat transfer modules compared to a pure boundary layer computation

the throat in comparison to the $1 \mathrm{D}$ expansion. Close to the nozzle exit, a lower near-wall layer velocity with respect to the 1D expansion occurs. This means that the gas composition in the near-wall layer is not determined by the local nozzle cross section as used for the $1 \mathrm{D}$ expansion. Thus, the $2 \mathrm{D}$ wall pressure distribution affects the boundary layer edge velocity as well as the corresponding gas composition, producing a significant impact on the resulting heat flux with respect to the $1 \mathrm{D}$ approach. The $2 \mathrm{D}$ flow is marked with a more intense acceleration downwards the throat section leading to rapid changes in gas composition. This effect slows down closer to the nozzle exit due to the restricted expansion capability.

The implementation of the above mentioned models atop of the selected boundary layer approach results in a more realistic heat flux description. Considering the whole thrust chamber (as presented in Fig. 6), 4 main domains can be identified.

Domain I is mainly characterized by the injection, eliminating as far as possible the impact of the boundary layer. The very thin boundary layer in this domain leads to a strong heat flux increase towards the injector head.

Domain II is only marked by the boundary layer, taking also into account the combustion efficiency.

The throat section domain III is mainly marked by the combustion efficiency. The shape of the heat flux curve in this domain is driven by the boundary layer and the cross-sectional change of the chamber.

The supersonic nozzle (domain IV) is marked by a rapid pressure change inducing a steep heat flux decrease towards the exit. The 2D approach results in a steeper slope after the throat followed by a weak bend - transition to a more flat decrease in the heat flux as compared to the 1D expansion. Such 
behavior is typical especially for a nonstreamline nozzle contour profiled using the Rao-method.

\section{METHOD VERIFICATION}

One major objective of developing the new computational tool is the simultaneous verification and validation using all available test data for recovering still existing shortcomings and determining the confidence level of the new method. Also, additional points were disclosed that is covering all necessary fields of applications in terms of heat transfer prediction.

With respect to the recent main focus of the development, the cross check activities were mainly dedicated to $\mathrm{LOx}-\mathrm{H}_{2}$ and $\mathrm{LOx}-\mathrm{CH}_{4}$, applying open- and closed-cycle thrust chamber configurations.

Figure 7 compares the measured and calculated heat transfer curves for a standard, segmented subscale configuration. The combination of the different models, considering injection, combustion efficiency, and boundary layer, results in a reasonably good prediction of the heat transfer level and distribution without additional adaptations.

Especially at the combustion chamber entrance, an acceptable heat flux evolution is obtained using the new RCFS-II code. The comparison with the Rocflam-II based combustion simulation further confirms the selected approach. Such analyses in defining the confidence level of the new version of the computational tool are very important for future full-scale design activities.

Figure 8 compares the heat transfer curves calculated using the Rocflam-II and RCFS-II codes for a $\mathrm{LOx}-\mathrm{LH}_{2}$ full-scale thrust chamber. The analysis of

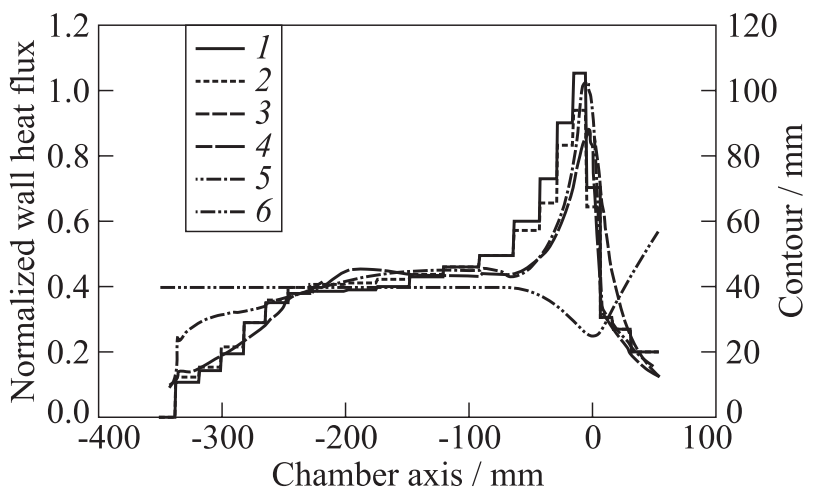

Figure 7 Comparison of heat transfer curves using subscale test data ( $\mathrm{LOx}^{-}$ hydrocarbon): 1 and 2 - heat fluxes 1 and 2 (measurements); 3 and 4 - RCFS-II $1 \mathrm{D}$ and 2D (new); 5 - Rocflam-RCFS-II; and 6 - contour 


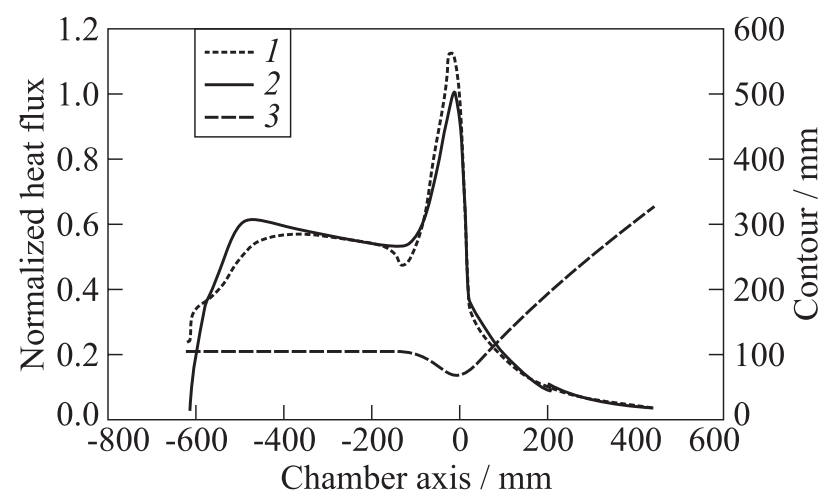

Figure 8 Heat transfer analysis on a full-scale TCA using Rocflam-II and RCFS-II codes (LOx-hydrogen): 1 - Rocflam-II/RCFS-II; 2 - RCFS-II, 2D; and 3-contour

the RCFS-II data shows a sutisfactory agreement between both methods which is necessary for the early phase of thrust chamber development.

The further emphasis of tool validation is, in particular, the analysis of closedcycle engine applications in view of future propulsion systems using $\mathrm{LOx}-\mathrm{H}_{2}$ and/or $\mathrm{LOx}_{-}-\mathrm{CH}_{4}$. Therefore, the available test data generated within the recent FLPP P8 test campaigns [8], applying closed-cycle schematics, will be also used to cross check the confidence of the upgraded engineering heat transfer code.

\section{CONCLUDING REMARKS}

The improved RCFS-II boundary-layer heat-transfer engineering tool presents a powerful option covering a wide range of applications in terms of propellant combination and operational conditions.

The main advantage of the new approach consists of the renouncement of adaptation factors to reflect a more realistic heat transfer picture on the hot gas side. The consideration of the effect of combustion efficiency on the overall heat flux distribution presents a major issue solved within the new tool. The new RCFS-II code also allows considering specific injection conditions relevant for open- and closed-cycle injection.

Based on still ongoing modifications and validations, a high accuracy in the prediction of heat flux evolution and level has been already demonstrated, without using any empirical data for adaptation and with increased user friendliness at the same time.

Nevertheless, further improvements are required for better reflecting all parameters relevant to heat transfer. The current development is mainly focused 
on in-house thrust chamber configurations and injection systems. The film layer modeling presents beside the amelioration of the injector model one major field of RCFS-II code improvement. This allows widening the field of application to small thrusters marked by the presence of film cooling rather than regenerative cooling.

The future development of the second-generation RCFS is not only addressed to improve the accuracy of hot gas side heat flux prediction. Further emphasis will be made on the improvement of the coolant side heat transfer modeling and prediction with respect to different cooling channel configurations and taking into account the impact of thermal stratification, curvature, as well as coolant circuit inlet and outlet effects.

\section{REFERENCES}

1. Fröhlich, A., M. Popp, G. Schmidt, and D. Thelemann. 1993. Heat transfer characteristics of $\mathrm{H}_{2} / \mathrm{O}_{2}$ - combustion chambers. AIAA Paper No. 93-1826.

2. Görgen, J., T. Aichner, and M. Frey. 2009. Spray combustion and heat transfer modelling in $\mathrm{LOx} / \mathrm{H}_{2}, \mathrm{LOx} / \mathrm{HC}$ and $\mathrm{MMH} / \mathrm{NTO}$ combustion chambers. 3rd European Conference for Aerospace Sciences (EUCASS). Versailles, France.

3. Knab, O., M. Frey, C. Maeding, K. Quering, D. Wiedmann, and W. Zeiss. 2009. Progress in combustion and heat transfer modelling in rocket thrust chamber applied engineering. AIAA Paper No. 2009-5477.

4. McBride, B., S. Gordon, and M. Reno. 1993. Coefficients for calculating thermodynamic and transport properties of individual species. NASA Technical Memorandum 4513.

5. Gordon, S., and B. McBride. 1994. Computer program for calculation of complex chemical equilibrium compositions and applications. NASA Reference Publication 1311.

6. Boudigues, S. 1987. Caracteristiques physiques et thermodynamiques de $\mathrm{N}_{2} \mathrm{O}_{4}$ et de MMH. Rapport technique No. 36/6112 EH, ONERA.

7. Dunn, S. S., D. E. Coats, G. R. Nickerson, and D. R. Berker. 1991. Two-dimensional kinetics (TDK91 PRO/X) nozzle performance computer programm. Carson City, Nevada: Software and engineering Associates Inc. Vols. I, II, III.

8. Strunz, R., F. Grauer, G. Hagemann, D. Preclik, X. Viot, G. Lé Forestier, and G. Dantu. 2008. Status of the $\mathrm{LOx} / \mathrm{GH}_{2}$ combustion activities within the furture launcher preporatory programme FLPP. Space Propulsion Conference. Heraklion, Greece. 